\title{
Correction to: Applying fuzzy AHP-TOPSIS technique in identifying the content strategy of sustainable manufacturing for food production
}

\section{Lanndon A. Ocampo ${ }^{1}$}

Published online: 18 June 2018

(C) Springer Nature B.V. 2018

\section{Correction to: Environ Dev Sustain}

$$
\text { https://doi.org/10.1007/s10668-018-0129-8 }
$$

Unfortunately, the below equations have been published incorrectly in the original publication. The correct equations are provided below:

Equation (11):

$$
X=\left(x_{i j}\right)_{m \times n}=\left(\sum_{k=1}^{K}\left(\omega_{k} * x_{i j}^{k}\right)\right)_{m \times n} .
$$

In Step 4 appearing above Eq. 13, the notation $v_{i j}$ is written twice. It should be read as:

Calculate the weighted normalized decision matrix $V=\left(v_{i j}\right)_{m \times n}$. The weighted normalized value $v_{i j}$ is calculated as follows:

$$
v_{i j}=r_{i j} \times w_{j},
$$

where $w_{j}$ is the weight of the $j$ th criterion or attribute defined by a pre-defined prioritization (weighting) process and $\sum_{j=1}^{n} w_{j}=1$.

Equations (14), (15), (16) and (17) should be read as:

$$
\begin{aligned}
& A^{+}=\left\{\left(\max _{i} v_{i j} \mid j \in C_{b}\right),\left(\min _{i} v_{i j} \mid j \in C_{c}\right)\right\}=\left\{v_{j}^{+} \mid j=1,2, \ldots, n\right\} \\
& A^{-}=\left\{\left(\min _{i} v_{i j} \mid j \in C_{b}\right),\left(\max _{i} v_{i j} \mid j \in C_{c}\right)\right\}=\left\{v_{j}^{-} \mid j=1,2, \ldots, n\right\},
\end{aligned}
$$

The original article can be found online at https://doi.org/10.1007/s10668-018-0129-8.

Lanndon A. Ocampo

lanndonocampo@gmail.com

1 Department of Industrial Engineering, Cebu Technological University, Corner M.J. Cuenco Ave.

\& R. Palma St., 6000 Cebu City, Philippines 
where $C_{b}$ and $C_{c}$ are the set of maximizing and minimizing criteria, respectively.

$$
\begin{aligned}
& S_{i}^{+}=\sqrt{\sum_{j=1}^{n}\left(v_{i j}-v_{j}^{+}\right)^{2}}, \quad i=1,2, \ldots, m \\
& S_{i}^{-}=\sqrt{\sum_{j=1}^{n}\left(v_{i j}-v_{j}^{-}\right)^{2}}, \quad i=1,2, \ldots, m .
\end{aligned}
$$

Correct Step 7, and Eq. (26) appearing below Table 7 in the original article should be read as:

7. The aggregate fuzzy numbers were then normalized into $\tilde{r}_{i j}$ using Eq. (26)

$$
\tilde{r}_{i j}=\left(\frac{X_{i j a}}{d_{j}^{*}}, \frac{X_{i j b}}{d_{j}^{*}}, \frac{X_{i j c}}{d_{j}^{*}}\right),
$$

where $d_{j}^{*}=\max _{c} X_{i j c}$.

Correct Steps 8 and 9 appearing below Table 7 must be read as below:

8. The fuzzy weighted normalized decision matrix $\tilde{V}=\left(\tilde{v}_{i j}\right)_{m \times n}$ is then found by multiplying the normalized aggregated fuzzy matrix $\tilde{R}=\left(\tilde{r}_{i j}\right)_{m \times n}$ and the global weight vector $(w)$ of decision options which is obtained in Step 5.

$$
\tilde{v}_{i j}=\tilde{r}_{i j} \times w_{j} .
$$

9. By using the vertex method, the separation distance is computed for each option from the fuzzy positive ideal solution $\left(\mathrm{A}^{+}\right)$and fuzzy negative ideal solution $\left(\mathrm{A}^{-}\right)$.

$$
\begin{gathered}
A^{+}=\left\{\tilde{v}_{1}^{+}, \tilde{v}_{2}^{+}, \tilde{v}_{3}^{+}, \ldots, \tilde{v}_{n}^{+}\right\} \\
A^{-}=\left\{\tilde{v}_{1}^{-}, \tilde{v}_{2}^{-}, \tilde{v}_{3}^{-}, \ldots, \tilde{v}_{n}^{-}\right\} \\
d\left(\tilde{v}_{i j}, \tilde{v}_{j}^{+}\right)=\sqrt{\frac{1}{3}\left[\left(\tilde{v}_{i j a}-\tilde{v}_{j a}^{+}\right)^{2}+\left(\tilde{v}_{i j b}-\tilde{v}_{j a}^{+}\right)^{2}+\left(\tilde{v}_{i j c}-\tilde{v}_{j a}^{+}\right)^{2}\right]} \\
d\left(\tilde{v}_{i j}, \tilde{v}_{j}^{-}\right)=\sqrt{\frac{1}{3}\left[\left(\tilde{v}_{i j a}-\tilde{v}_{j a}^{-}\right)^{2}+\left(\tilde{v}_{i j b}-\tilde{v}_{j a}^{-}\right)^{2}+\left(\tilde{v}_{i j c}-\tilde{v}_{j a}^{-}\right)^{2}\right]} \\
d_{i}^{+}=\sum_{j=1}^{n} d\left(\tilde{v}_{i j}, \tilde{v}_{j}^{+}\right) \\
d_{i}^{-}=\sum_{j=1}^{n} d\left(\tilde{v}_{i j}, \tilde{v}_{j}^{-}\right) .
\end{gathered}
$$

